\title{
Pseudo-Analytical Modeling of Tilted-Coil Antennas in Anisotropic Geophysical Formations
}

\author{
Guilherme S. Rosa and José R. Bergmann, \\ Center for Telecommunications Studies, Pontifical Catholic University of Rio de Janeiro, \\ Rio de Janeiro, RJ 22451-900,Brazil, \{guilhermesimondarosa, bergmann\}@cetuc.puc-rio.br \\ Fernando L. Teixeira \\ ElectroScience Laboratory, The Ohio State University, Columbus, OH 43212,USA, teixeira.5@osu.edu
}

\begin{abstract}
In this paper we present an efficient mode-matching technique to analyze tilted-coil antennas in anisotropic geophysical formations. In this problem, a number of coil antennas with arbitrary relative tilt angle with respect to the symmetry axis are used to radiate electromagnetic fields in a cylindrically layered medium comprised of a metallic mandrel, a borehole, and a surrounding layered Earth formation. This configuration corresponds to that of directional well-logging tools used in oil and gas exploration. Our technique combines closed-form solutions for the Maxwell's Equations in uniaxially anisotropic and radiallystratified cylindrical coordinates with the generalized scattering matrix (GSM) at each axial discontinuity based on the modematching technique. The field from the transmitter tilted-coil source is represented by a set of modal coefficients which, after computation using GSM matrices, are used to compute the transimpedances. We present validation results which show that our technique can efficiently model directional well-logging tools used for oil and gas exploration.
\end{abstract}

Index Terms - Anisotropic media, mode matching methods, stratified media, well logging tools.

\section{INTRODUCTION}

Logging-while-drilling (LWD) and measurement-while-drilling (MWD) tools are frequently used to evaluate hydrocarbon reservoirs. The complex geophysical formations present in this type of problem can be successfully modeled using numerical techniques such as finite-differences (FD), finite elements (FE) and finite volumes (FV) [1]-[5]. However, these brute-force techniques suffer from relatively high cost in terms of both computer memory and CPU time. Bearing in mind that oil exploration usually employs LWD tools comprised of coil antennas wrapped around a metallic mandrel inside a borehole, as illustrated in Fig. 1, a series of practical scenarios can be properly approximate as a radially-stratified medium [6]. In this case, efficient algorithms based on pseudoanalytical methods [6]-[10] are good alternatives to model these geometries and provide efficient inversion algorithms designed to estimate the Earth formation properties given the tool responses. More complicated geometries including inhomogeneities in the Earth formation along both radial and axial directions can be accounted by using the numerical mode-matching (NMM), which may be seen 


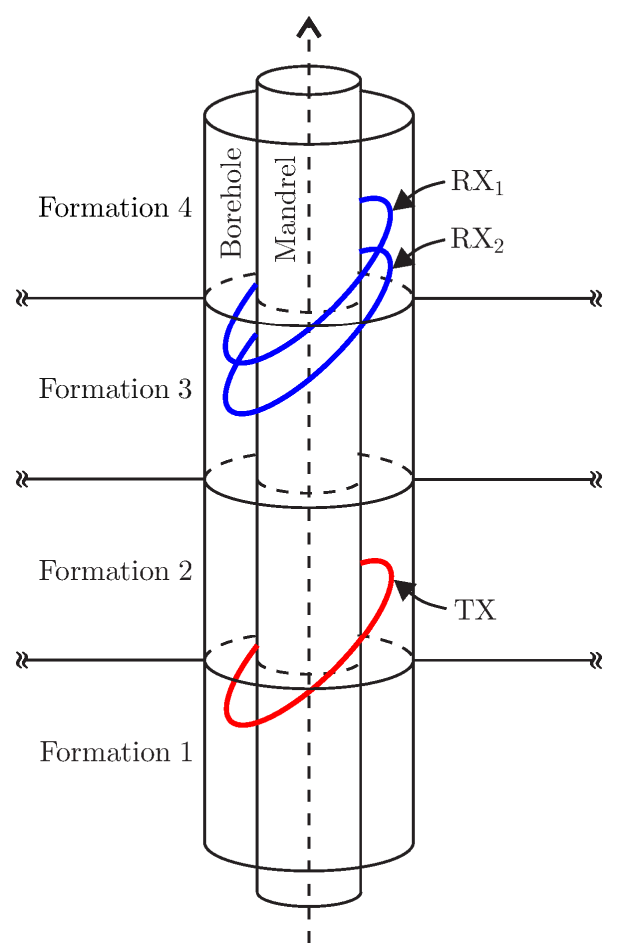

Fig. 1. Geometry of a triaxial TCA well-loogging tool within a stratified geophysical formation.

as providing a middle-ground in terms of computational costs between brute-force and pseudoanalytical techniques [11]-[14]. The NMM is a very accurate approach that combines the FD or FE method in one direction with an analytical (modal) expansion in the other directions.

Tilted-coil antennas (TCA) have been introduced for well-logging tools [7]-[9] to provide azimuthal sensitivity. In [14], the NMM was extended to model triaxial TCA tools in vertical wells with both radial and axial stratifications using a vertical mode expansion in conjunction with a horizontal mode-matching. The NMM can be also formulated in an alternate fashion, i.e., with a horizontal mode expansion [10]-[13]. However, since TCA has a non-zero span along the axial direction, the vertical mode expansion is more appropriate to this type of problem. Although suitable to model TCA tools in straight wells, the approach used in [14] cannot be easily generalized to curved wells where axial bending is also present. These wells occur during deviated drilling and, in this case, the axial (vertical) mode-matching is a more natural choice.

In this work, we employ an axial mode-matching combining attractive features of the early described pseudo-analytical techniques, to obtain a flexible technique for analyzing directional welllogging tools in anisotropic formations which can be easily extended to model wells with curvature.

\section{Pseudo-Analytical Formulation}

The geometry shown in Fig. 1 is used to model different sections of the regions around the welllogging tool as a bounded, radially-stratified waveguide. We can decompose the radiated field into axial (to $z$ ) and transversal components, and the subscripts $z$ and $s$, respectively, are used to assign those directions. Our media is characterized by the complex permeability $\overline{\bar{\mu}}=\operatorname{diag}\left(\mu_{s}, \mu_{s}, \mu_{z}\right)$ and permissibility $\overline{\bar{\epsilon}}=\operatorname{diag}\left(\epsilon_{s}, \epsilon_{s}, \epsilon_{z}\right)$ tensors in cylindrical coordinates. Field solutions for Maxwell's 
equations in uniaxially anisotropic multilayered cylindrical structures are well-known, and to avoid repetition, we adopt here a notation similar to that in [2], [7], [9], [13]-[15] by assuming a timeharmonic dependence in the form $\exp (-i \omega t)$. A brief theoretical background of the formulation will be presented in this section. More mathematical details can be found in [16].

\section{A. Electromagnetic Fields Along Radial Stratifications}

The forward propagating axial fields can be written in a compact fashion as

$$
\left[\begin{array}{l}
E_{z} \\
H_{z}
\end{array}\right]=\sum_{n=-\infty}^{\infty} \sum_{p=1}^{\infty}\left[\begin{array}{l}
e_{z, n p}(\rho) \\
h_{z, n p}(\rho)
\end{array}\right] e^{i n \phi+i k_{z, n p} z}
$$

The modal propagation constant in the $z$ direction is $k_{z, n p}$, and the radial propagation constant $k_{\rho, n p}$ satisfies

$$
k_{\rho, n p}^{2}=k_{s}^{2}-k_{z, n p}^{2}, \quad k_{s}^{2}=\omega^{2} \mu_{s} \epsilon_{s} .
$$

In order to simplify the notation, we will temporarily drop the modal subscript $n p$ and also the argument of $e_{z, n p}$ and $h_{z, n p}$, restoring them later as needed. The radially-dependent coefficients in (1) are given by a linear combination of first kind Hankel and Bessel function of integer order $n$, as given in [15], [16]. The transversal field components can be expressed as a combination of the axial ones using well-known relations for cylindrical coordinates [13].

Consider the radially stratified waveguide shown in Fig. 2, composed by $N$ layers. Each layer is formed by an uniaxially anisotropic medium over $r_{j-1}<\rho<r_{j}, j=1,2, \ldots, N$, and for $0<\phi<2 \pi$. The fields at each layer $j$ can be written, in a shorthand notation, as

$$
\begin{gathered}
\bar{g}_{j \alpha}=\left[\overline{\bar{H}}_{\alpha n}\left(k_{j \rho} r_{j}\right)+\overline{\bar{J}}_{\alpha n}\left(k_{j \rho} r_{j}\right) \tilde{\overline{\bar{R}}}_{j, j+1}\right] \bar{a}_{j}, \text { or } \\
\bar{g}_{j \alpha}=\left[\overline{\bar{H}}_{\alpha n}\left(k_{j \rho} r_{j}\right) \tilde{\overline{\bar{R}}}_{j, j-1}+\overline{\bar{J}}_{\alpha n}\left(k_{j \rho} r_{j}\right)\right] \bar{b}_{j},
\end{gathered}
$$

where $\bar{g}_{j \alpha}=\left[\begin{array}{ll}e_{j \alpha} & h_{j \alpha}\end{array}\right]^{t}$ is a 2-by-1 column vector with the electric and magnetic non-harmonic portion of the fields components in direction $\alpha=\{\rho, \phi, z\}$. Notice that due the translational and azimuthal invariance of the problem; see [13, pp. 163-164]; both $z$ and $\phi$ dependences can be suppressed in (1). In order to enforce the appropriated boundary conditions at $\rho=r_{j}$, we employ 2-

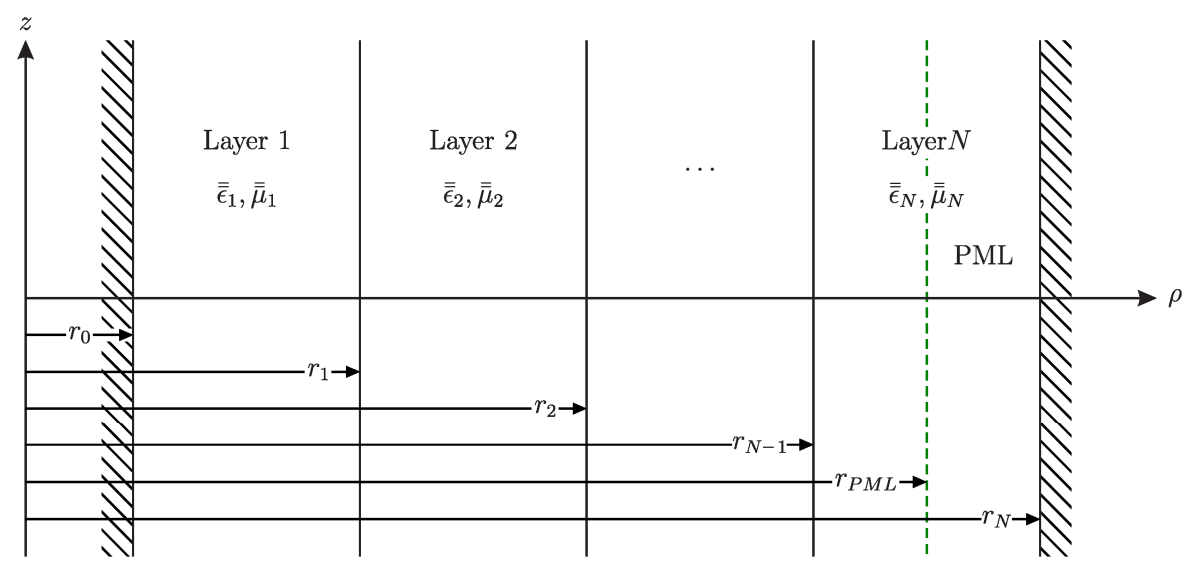

Fig. 2. Longitudinal and cross-sectional views of a radially-stratified waveguide. Perfect electric conductors are indicated by the stripes. 
by-2 generalized reflection matrices $\tilde{\overline{\bar{R}}}_{j, j \pm 1}$ as in [13, Chap. 3], [15]. We can find the amplitudes of adjacent layers to layer $j$ using

$$
\bar{a}_{j+1}=\overline{\bar{S}}_{j, j+1} \bar{a}_{j}, \quad \text { and } \quad \bar{b}_{j-1}=\overline{\bar{S}}_{j, j-1} \bar{b}_{j}
$$

where $\overline{\bar{S}}_{j, j \pm 1}$ is defined in [13].

Using (5) in (3) and (4), it follows

$$
\left(\overline{\bar{I}}-\tilde{\overline{\bar{R}}}_{j, j+1} \tilde{\overline{\bar{R}}}_{j, j-1}\right) \bar{b}_{j}=\left(\overline{\bar{I}}-\tilde{\overline{\bar{R}}}_{j, j-1} \tilde{\overline{\bar{R}}}_{j, j+1}\right) \bar{a}_{j}=\overline{0},
$$

which allow us to find the guidance condition for the modes as $\operatorname{det}\left(\overline{\bar{I}}-\tilde{\overline{\bar{R}}}_{j, j+1} \tilde{\overline{\bar{R}}}_{j, j-1}\right)=0$. Since $\tilde{\overline{\bar{R}}}_{N, N+1}=\overline{\bar{R}}_{N, N+1}$ [13], by setting $\overline{\bar{M}}=\overline{\bar{I}}-\overline{\bar{R}}_{N, N+1} \tilde{\overline{\bar{R}}}_{N, N-1}$, the eigenvalue solutions of characteristic equation

$$
\operatorname{det}(\overline{\bar{M}})=0
$$

are the discrete values of $k_{z}$ that contribute to our modal solution. We can next find all desired eigensolutions of (7) in a given region of the complex plane $k_{z}$ (or $k_{j \rho}$ ) using the Argument Principle [17], [18] and the technique shown in [19].

Instead of matching the source jump condition to derive the eigenmode amplitudes as in [7], [9], [14], here we prefer find a source-free solution and then include the excitation apart. We can easily verify that solutions to the homogeneous linear system $\overline{\bar{M}} \bar{b}_{N}=\overline{0}$ correspond to the null space of $\overline{\bar{M}}$, i.e., $\bar{b}_{N}=\operatorname{null}(\overline{\bar{M}})$. Now we have the modal amplitudes for the outermost radial standing wave, the fields in all layers can be derived recursively using (5) and (4).

In order to mimic an unbounded medium, the radial direction is truncated by a perfectly matched layer (PML) [20], [21]. The PML extends over $r_{P M L}<\rho<r_{N}$, as illustrated in Fig. 2, and we use the complex coordinate stretching formulation of the PML because it allow us to reuse all close-form eigenmode formulas shown above, such we just need to select an appropriated complex-value for the outermost radius of the waveguide [21]-[23], i.e., $r_{N} \rightarrow \tilde{r}_{N}=r_{N}+i \alpha_{P M L}$.

\section{B. Electromagnetic Fields Along Axial Stratifications}

The previous results can be applied to analyze an axially infinitely-long cylindrical waveguide. To properly model the axial discontinuities shown in Fig. 1, we need to match the boundary condition at each waveguide junction. Now, looking at equation (1), we can anticipate that the $n$th modal field of region $j$ will couple with only the $(-n)$ th field of the adjacent regions $j \pm 1$. This is due to the orthogonality of the azimuthal harmonics and allows us to substantially simplify our analysis.

By imposing the conservation of reaction [24], [25] of the fields in the common aperture (at $z=z_{1}$ in Fig. 3) between the regions 1 and 2, we can relate the forward and backward modal amplitudes in each waveguide as

$$
\left[\begin{array}{l}
\bar{A}_{1}^{-} \\
\bar{A}_{2}^{+}
\end{array}\right]=\left[\begin{array}{ll}
\overline{\bar{R}}_{12} & \overline{\bar{T}}_{21} \\
\overline{\bar{T}}_{12} & \overline{\bar{R}}_{21}
\end{array}\right]\left[\begin{array}{l}
\bar{A}_{1}^{+} \\
\bar{A}_{2}^{-}
\end{array}\right],
$$




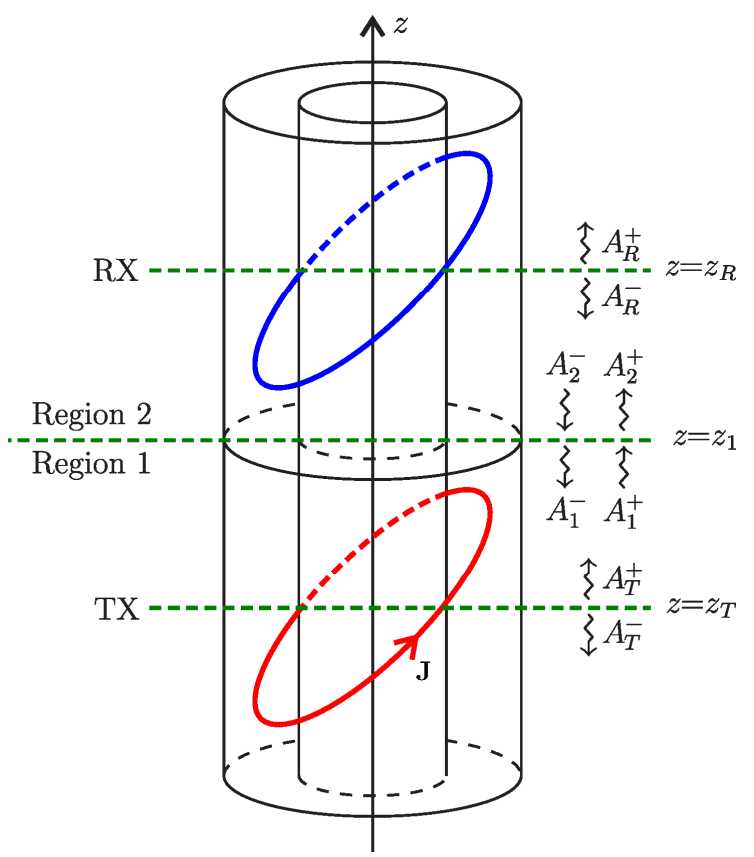

Fig. 3. Junction between two radially-stratified waveguides.

where the generalized scattering matrix (GSM) comprises

$$
\begin{aligned}
& \overline{\bar{R}}_{12}=\left(\overline{\bar{Q}}_{1}+\overline{\bar{X}}_{12}^{t} \bar{Q}_{2}^{-1} \overline{\bar{X}}_{12}\right)^{-1}\left(\overline{\bar{Q}}_{1}-\overline{\bar{X}}_{12}^{t} \overline{\bar{Q}}_{2}^{-1} \overline{\bar{X}}_{12}\right), \\
& \overline{\bar{T}}_{21}=2\left(\overline{\bar{Q}}_{1}+\overline{\bar{X}}_{12}^{t} \overline{\bar{Q}}_{2}^{-1} \overline{\bar{X}}_{12}\right)^{-1} \overline{\bar{X}}_{12}^{t}, \\
& \overline{\bar{T}}_{12}=2\left(\overline{\bar{Q}}_{2}+\overline{\bar{X}}_{12} \overline{\bar{Q}}_{1}^{-1} \overline{\bar{X}}_{12}^{t}\right)^{-1} \overline{\bar{X}}_{12}, \text { and } \\
& \overline{\bar{R}}_{21}=-\left(\overline{\bar{Q}}_{2}+\overline{\bar{X}}_{12} \overline{\bar{Q}}_{1}^{-1} \overline{\bar{X}}_{12}^{t}\right)^{-1}\left(\overline{\bar{Q}}_{2}-\overline{\bar{X}}_{12} \overline{\bar{Q}}_{1}^{-1} \overline{\bar{X}}_{12}^{t}\right) .
\end{aligned}
$$

The entries of matrices $\overline{\bar{Q}}_{1}, \overline{\bar{Q}}_{2}$ and $\overline{\bar{X}}_{12}$ are closely related with the self-reaction of fields in region 1 and 2, and to the reaction of fields in region 1 to region 2 , such as

$$
\begin{gathered}
\left.\overline{\bar{X}}_{12}\right|_{p^{\prime}, p}=X_{1(n p), 2\left(-n p^{\prime}\right)}, \\
\left.\overline{\bar{Q}}_{j}\right|_{p^{\prime}, p}=\delta_{p, p^{\prime}} X_{j(n p), j\left(-n p^{\prime}\right)},
\end{gathered}
$$

where $\delta_{p, p^{\prime}}$ denotes the Kronecker delta, and the reaction integrals are defined in terms of the fields in regions $i$ and $j$ as

$$
X_{i(n p), j\left(-n p^{\prime}\right)}=-2 \pi \int_{r_{0}}^{\tilde{r}_{N}}\left(e_{i \rho, n p} h_{j \phi, n p^{\prime}}+e_{i \phi, n p} h_{j \rho, n p^{\prime}}\right) \rho d \rho .
$$

We are able to obtain analytical results for the above coupling integral as in [16], [22], [26].

The above procedure can be applied to all remaining axial discontinuities, allowing us to get one GSM to each axial discontinuity.

\section{Mode Excitation from a TCA Source}

Consider a transmitter TCA with electrical current density given by

$$
\mathbf{J}=I_{T} \delta\left(\rho-\rho_{T}\right) \delta\left(z-z_{T}+\rho_{T} \tan \theta_{T} \cos \left(\phi-\phi_{T}\right)\right)\left(\hat{\phi}+\hat{z} \tan \theta_{T} \sin \left(\phi-\phi_{T}\right)\right),
$$

where $I_{T}$ is the current source amplitude, $\rho_{T}$ and $z_{T}$ are the radial and axial position of the coil, and $\phi_{T}$ and $\theta_{T}$ are the azimuthal and elevation tilt angles [7] as shown in Fig. 3 and Fig. 4a. 


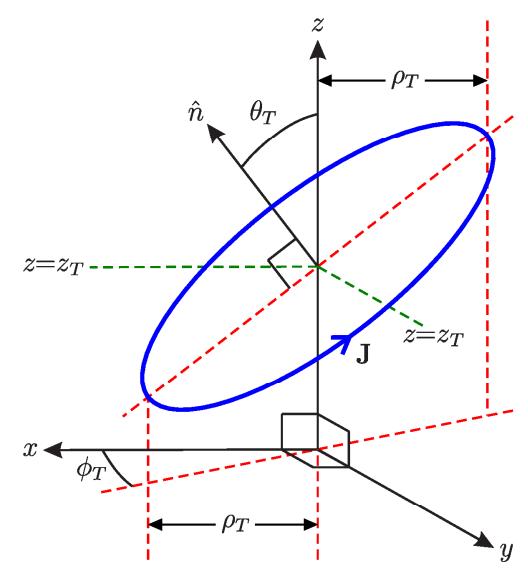

(a)

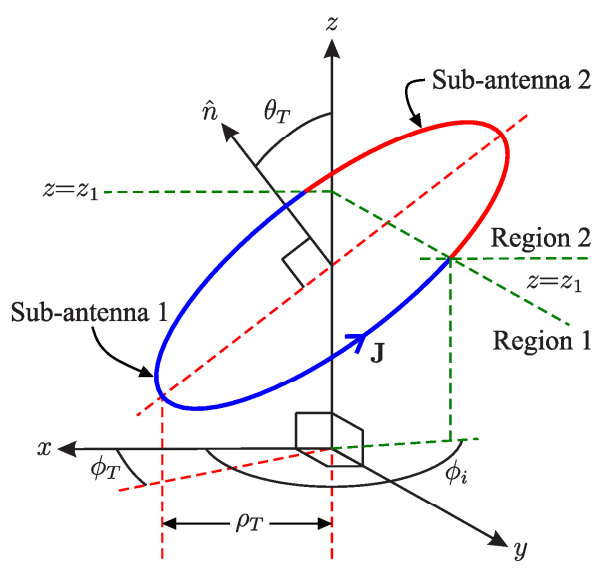

(b)

Fig. 4. Tilted-coil antenna with electrical current density $\mathbf{J}$ completely inside one axial region is represented in (a).

The proposed TCA segmentation for the source crossing two axial regions at $z=z_{1}$ is shown in (b).

The electromagnetic field radiated by such transmitter TCA can be expressed in terms of the waveguide modes as

$$
\begin{aligned}
\mathbf{E}^{ \pm} & =\sum_{n=-\infty}^{\infty} \sum_{p=1}^{\infty} A_{T, n p}^{ \pm}\left[\mathbf{e}_{s, n p}(\rho) \pm \hat{z} e_{z, n p}(\rho)\right] e^{ \pm i k_{z, n p}\left(z-z_{T}\right)+i n \phi}, \\
\mathbf{H}^{ \pm} & =\sum_{n=-\infty}^{\infty} \sum_{p=1}^{\infty} A_{T, n p}^{ \pm}\left[ \pm \mathbf{h}_{s, n p}(\rho)+\hat{z} h_{z, n p}(\rho)\right] e^{ \pm i k_{z, n p}\left(z-z_{T}\right)+i n \phi},
\end{aligned}
$$

for $z \gtrless z_{T} \pm \rho_{T} \tan \theta_{T}$. By using the Lorentz reciprocity theorem [27, p. 41], we can determine the unknown modal amplitude $A_{T, n p}^{ \pm}$as

$$
A_{T, n p}^{ \pm}=\frac{S_{n p}^{ \pm}}{N_{n p}}
$$

where the source excitation

$$
S_{n p}^{ \pm}=I_{T} \rho_{T}\left[e_{\phi, n p}\left(\rho_{T}\right) I_{1}\left(-n, \pm \kappa_{T}\right) \pm e_{z, n p}\left(\rho_{T}\right) \tan \theta_{T} I_{2}\left(-n, \pm \kappa_{T}\right)\right] e^{\mp i k_{z, n p} z_{T}}
$$

is normalized by $N_{n p}=-2 X_{j(n p), j(-n p)}$. In the above, we consider that the source is inside the region $j$, and the appropriated radial layer (of region $j$ ) must be selected based on the value of $\rho_{T}$, and $\kappa_{T}=\rho_{T} \tan \theta_{T} k_{z, n p}$. Furthermore, $I_{1}$ and $I_{2}$ correspond to simple integrals over the azimuthal angle given by

$$
I_{1}(n, \kappa)=\int_{-\pi}^{\pi} e^{i n \phi+i \kappa \cos \phi} d \phi, \quad \text { and } \quad I_{2}(n, \kappa)=\int_{-\pi}^{\pi} \sin \phi e^{i n \phi+i \kappa \cos \phi} d \phi,
$$

which can be solved in closed-form [7]. As a further simplification, we can show that our initial series solution in (17) and (18) reduces to a cosine series for positive integer $n$ as shown in [16].

By combining the source amplitudes $A_{T, n p}^{ \pm}$with the GSM matrices, the modal amplitudes $A_{R, n p}^{ \pm}$ can be obtained at an observation point on the plane $z=z_{R}$, as shown in Fig. 3. After a few simplifications, the receiver voltage (e.m.f.) can be obtained by performing a line integral of the electric field along the receiver TCA. 
In this analysis, we consider that the TCAs are completely inside one axial region. If that is not the case, we can partition the TCA into segments associated to each region, calculate the contribution of each segment individually, as suggested in representation shown in Fig. 4b, and use the superposition to find the total radiated field. In this way, we must split the integration over $\phi$ into (21) by means of an appropriated value of $\phi_{i}$ as show in Fig. 4b. These integrals do not compute in closed-form but a suitable fast convergent series representation exists based on the Jacobi-Anger expansion [28, p. 361].

\section{NUMERICAL RESULTS}

\section{A. Validation}

To illustrate the application of the proposed method, we present simulations of a triaxial logging tool consisting of one transmitter and two receivers TCAs in a vertical-well borehole traversing an anisotropic Earth formation with two horizontal layers. The problem includes a 4 -in-radius metallic mandrel, in which the TCAs are placed around, at the fixed radial distance $\rho_{T, R}=4.5 \mathrm{in}$. We consider a 5 -in-radius borehole filled with oil-based mud having the isotropic conductivity $\sigma=5 \times 10^{-4} \mathrm{~S} / \mathrm{m}$. Surrounding the borehole, the horizontal and vertical component of the conductivity of the bottom formation are 5 and $1 \mathrm{~S} / \mathrm{m}$. The top formation is characterized by 1 and $5 \mathrm{~S} / \mathrm{m}$, respectively. The axial positions of the transmitter, and receivers $\mathrm{RX}_{2}$ and $\mathrm{RX}_{1}$ (see Fig. 1) are $z_{T}, z_{T}+24$ in and $z_{T}+30$ in, respectively. This geometry was considered before in [2], [8] and is used here to validate our formulation. For more details on this geometry, see [2], [8]. We truncate the radial domain at $60 \mathrm{in}$, at about four times the skin depth of the les conductive layer. We include all modes whose axial attenuation is less than $60 \mathrm{~dB}$ at 5 in, and have considered only azimuthal indexes $n=0$ and $n=1$. The high order azimuthal harmonics give negligible contribution to total solution.

Fig. 5 and Fig. 8 plot the voltage due to a unit current excitation $\left(I_{T}=1 \mathrm{~A}\right.$ at $\left.2 \mathrm{MHz}\right)$ at the receivers antennas $\mathrm{RX}_{1}$ and $\mathrm{RX}_{2}$ as a function of the axial position $z_{T}$ of the tool, considering a transmitter TCA with fixed tilt angle of $45^{\circ}$, and four different receiver TCA tilt angles: $\theta_{R}=15^{\circ}, 25^{\circ}, 35^{\circ}$ and $45^{\circ}$. Very good agreement is observed versus the results from [2]

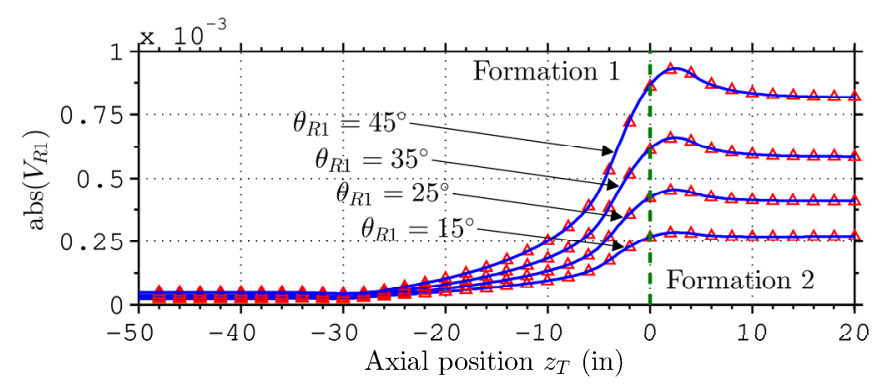

Fig. 5. Voltage amplitude at TCA receiver $\mathrm{RX}_{1}$. The results from the present approach are shown by solid lines. The small triangles are results from [2]. 


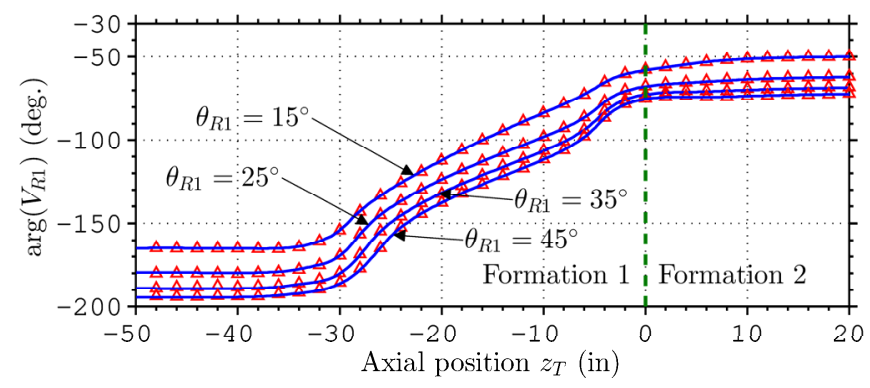

Fig. 6. Voltage phase at TCA receiver $\mathrm{RX}_{1}$. The results from the present approach are shown by solid lines. The small triangles are results from [2].

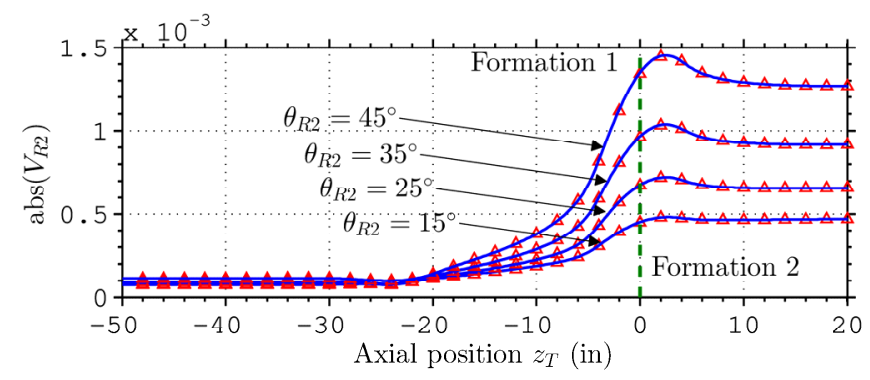

Fig. 7. Voltage amplitude at TCA receiver $\mathrm{RX}_{2}$. The results from the present approach are shown by solid lines. The small triangles are results from [2].

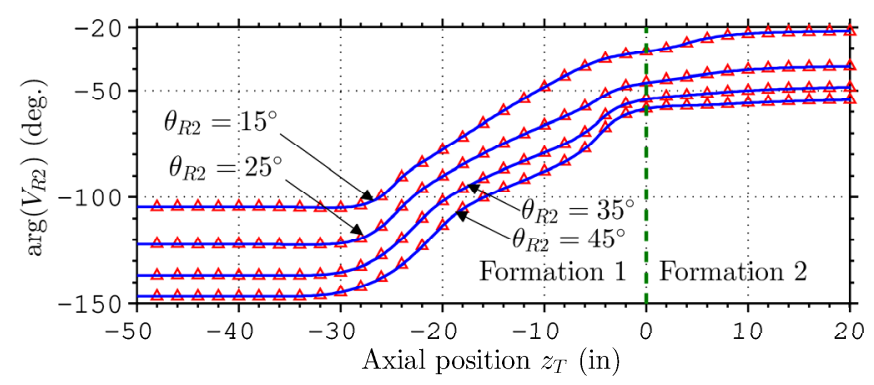

Fig. 8. Voltage phase at TCA receiver $\mathrm{RX}_{2}$. The results from the present approach are shown by solid lines. The small triangles are results from [2].

\section{B. Modelling TCA Antennas inside Mandrel Grooves}

After validating our new pseudo-analytical formulation, we now analyze the effect of a more realistic source excitation. Instead of install the TCA antennas around the mandrel, we consider the antennas now are wounded inside grooves on the mandrel pipe, as illustrated in Fig. 9. The mandrel presents a radius of 4 in, and the indentations are 2 -in-depth in radial direction. The TCA antennas are placed at $\rho=3 \mathrm{in}$. The axial position of the antenna TX is on middle of a 8 -in-groove, as shown in Fig. 9. The receivers TCA antennas $\mathrm{RX}_{1}$ and $\mathrm{RX}_{2}$ are placed inside a 14 -in-groove, such these receivers are 30 -in and 24 -in far from the transmitter. We consider a borehole filled with oil-based mud having the isotropic conductivity $\sigma=5 \times 10^{-4} \mathrm{~S} / \mathrm{m}$. The surrounding soil formation is an anisotropic media, and its horizontal and vertical component of the conductivity are 5 and $1 \mathrm{~S} / \mathrm{m}$, respectively. Also, we consider that all antennas are azimuthally aligned, i.e., $\phi_{T}=\phi_{R 1}=\phi_{R 2}$. Assuming a unit current excitation at $2 \mathrm{MHz}$, the tilt angle of each TCA antenna will be investigated in the following. We truncate the radial domain at $60 \mathrm{in}$, and have include all modes whose axial 

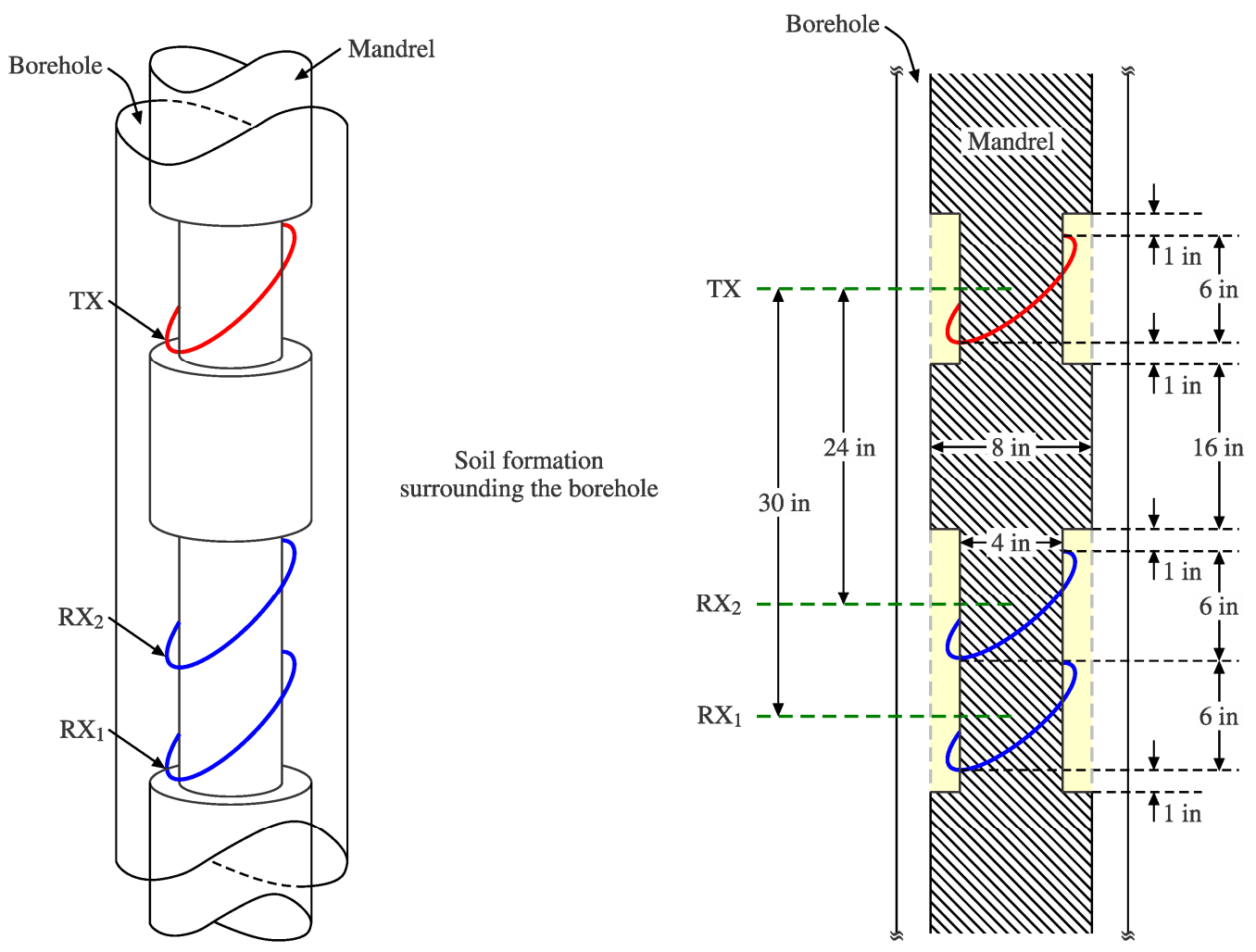

Fig. 9. Model employed to simulate the mandrel indentations. The details of the antennas positions and the dimensions of grooves on mandrel are shown on the right, in which the TCAs have $\rho_{T}=\rho_{R 1}=\rho_{R 2}=3$ in.

attenuation is less than $30 \mathrm{~dB}$ at 1 in; a very conservative criteria. Again, only azimuthal indexes $n=0$ and 1 are included because the contribution of the high order ones are negligible.

Fig. 10 shows results for the voltage at TCA receiver $\mathrm{RX}_{1}$ for various values of $\theta_{T}$ and $\theta_{R 1}$, in which the tilt angles vary from $0^{\circ}$ to $45^{\circ}$. Similarly, Fig. 11 presents the voltage at $\mathrm{RX}_{2}$ for several tilt angles associated to this receiver antenna. We can see that as the tilt angle of TCAs increases, the received voltage becomes higher as well.
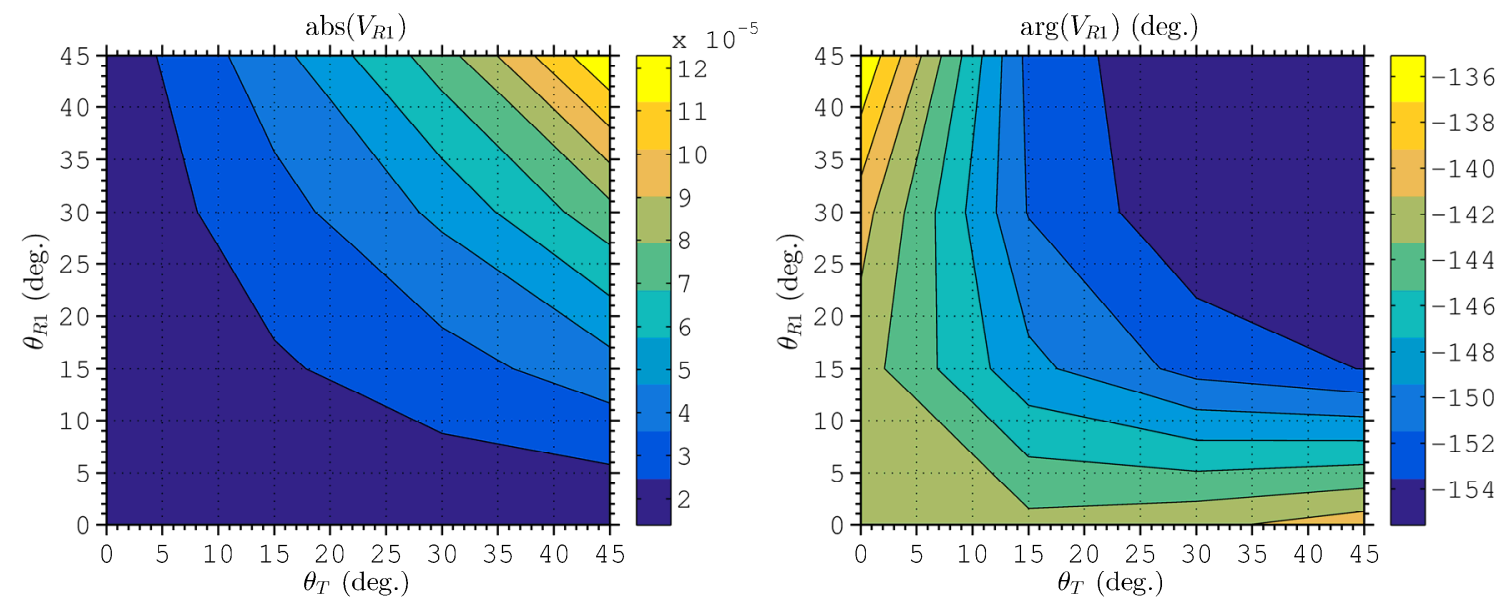

Fig. 10. Voltage at TCA receiver $\mathrm{RX}_{1}$ for various values of $\theta_{T}$ and $\theta_{R 1}$. 

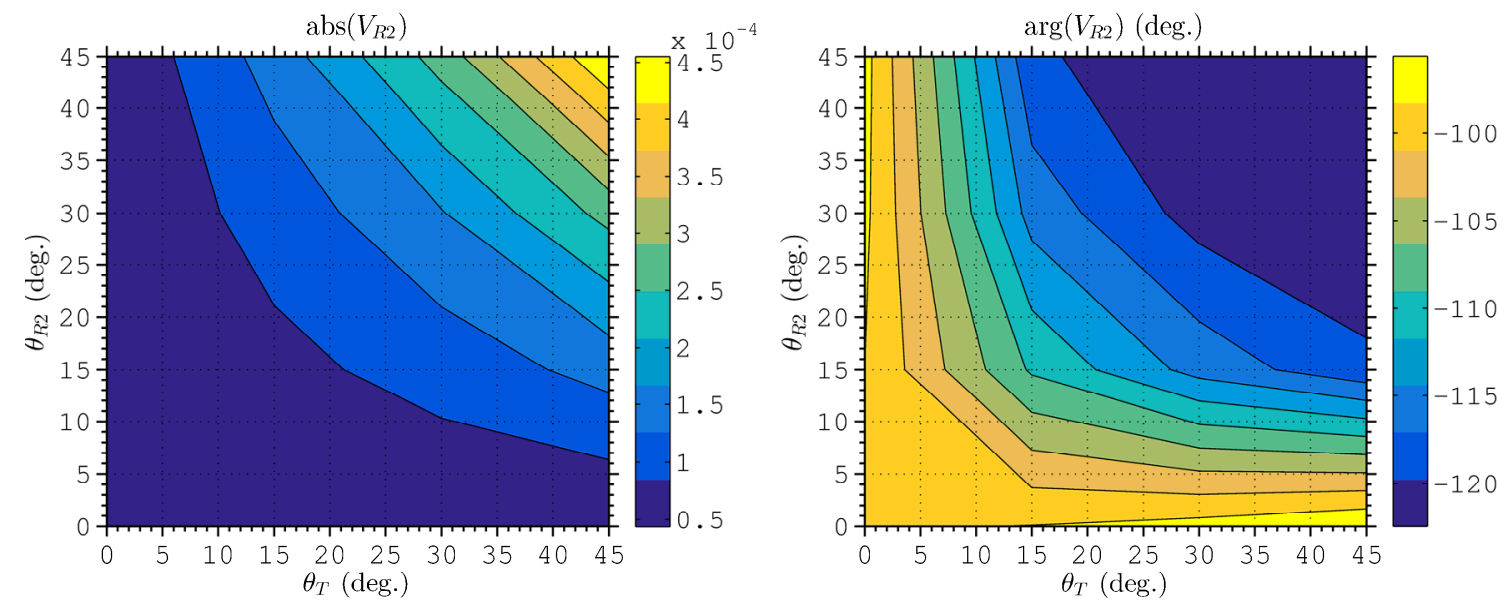

Fig. 11. Voltage at TCA receiver $\mathrm{RX}_{2}$ for various values of $\theta_{T}$ and $\theta_{R 2}$.

In geophysical prospection using LWD tools, the parameters of interest are the amplitude ratio $(A R)$ and phase difference $(P D)$ between the voltages at two antennas [2]:

$$
\begin{gathered}
A R=20 \log _{10}\left(V_{R 2} / V_{R 1}\right), \\
P D=\arg \left(V_{R 2}\right)-\arg \left(V_{R 1}\right) .
\end{gathered}
$$

Proper combination of the results showed above in Fig. 10 and Fig. 11 allow us to describe $A R$ and $P D$ responses for a desired LWD tool under investigation. Considering fixed transmitter TCA tilt angles $\theta_{T}=0^{\circ}, 15^{\circ}, 30^{\circ}$ and $45^{\circ}$, we can derive the results shown in Fig. 12-Fig. 15.

For $\theta_{T}=0^{\circ}$, only fields associated to azimuthal order $n=0$ are excited, such $A R$ and $P D$ due tilts in receivers observed in Fig. 12 cover the range of $8.4 \mathrm{~dB}$ to $9.8 \mathrm{~dB}$ and $37^{\circ}$ to $46^{\circ}$, respectively. As said before, for $\theta_{T}>0^{\circ}$, fields with $n=0$ and $n=1$ represent the main contribute to received voltage. In Fig. 13 we can clearly see the effects of fields with azimuthal index $n=1$ when $\theta_{T}=15^{\circ}$ : now the variations of $A R$ and $P D$ are over $1 \mathrm{~dB}$ to $17 \mathrm{~dB}$ and $23^{\circ}$ to $55^{\circ}$. This range of variation increase with $\theta_{T}$, as illustrated in Fig. 14 (for $\theta_{T}=30^{\circ}$ ) and Fig. 15 (for $\theta_{T}=45^{\circ}$ ). Notably, in these two cases we can observe negative values for $A R$; i.e, for some combinations of $\theta_{R 1}$ and $\theta_{R 2}$ the voltage received by $\mathrm{RX}_{1}$ is larger than those in $\mathrm{RX}_{2}$ (which is closer to TX).
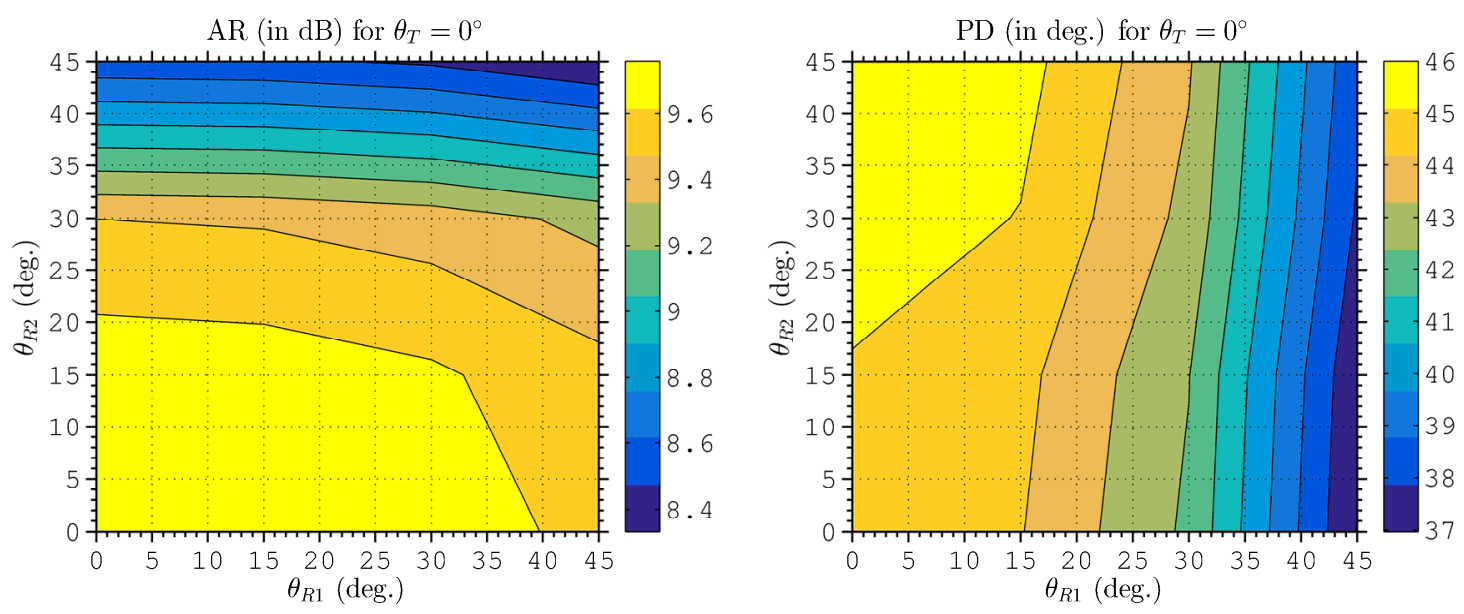

Fig. 12. Amplitude ratio and phase difference response for a transmitter TCA with $\theta_{T}=0^{\circ}$ in respect to the tilt angle of receivers. 

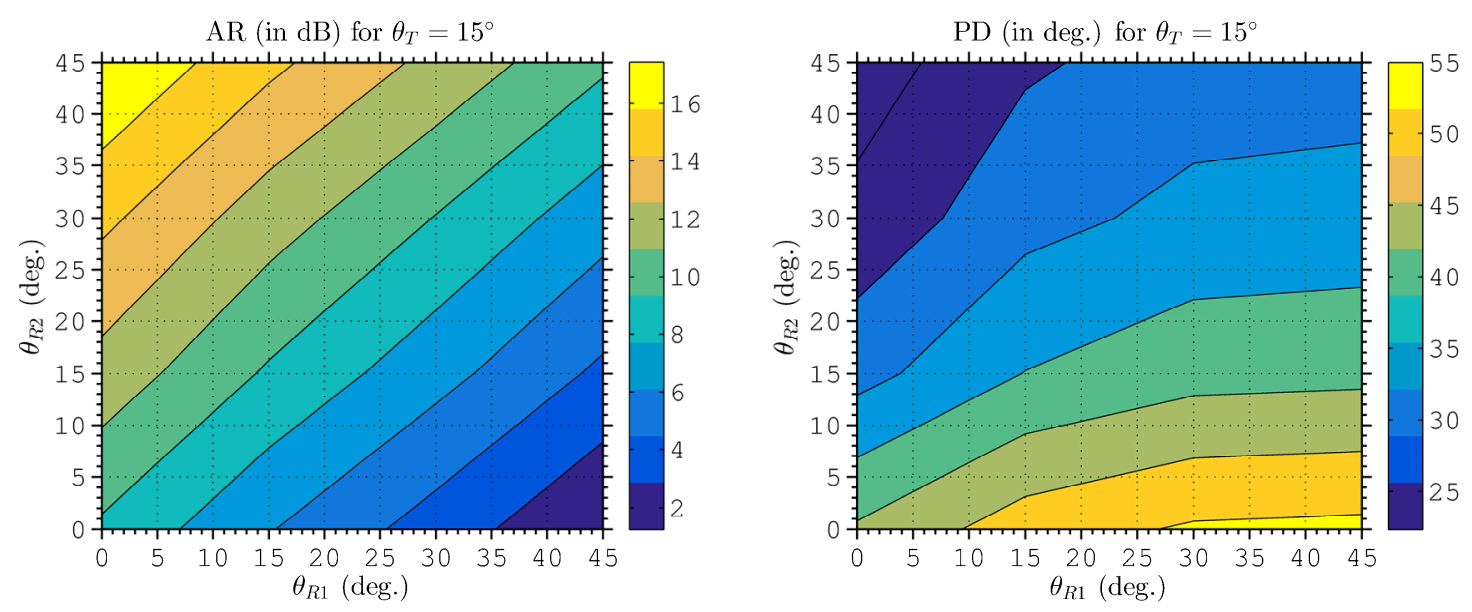

Fig. 13. Amplitude ratio and phase difference response for a transmitter TCA with $\theta_{T}=15^{\circ}$ in respect to the tilt angle of receivers.
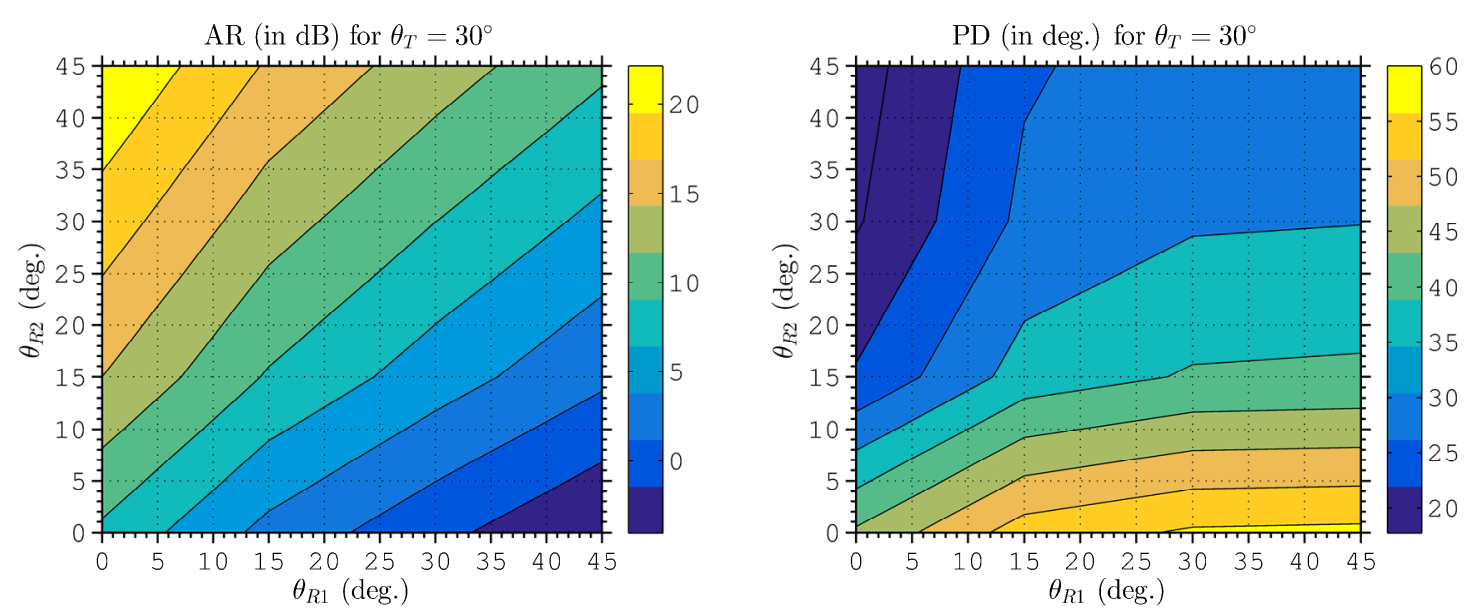

Fig. 14. Amplitude ratio and phase difference response for a transmitter TCA with $\theta_{T}=30^{\circ}$ in respect to the tilt angle of receivers.
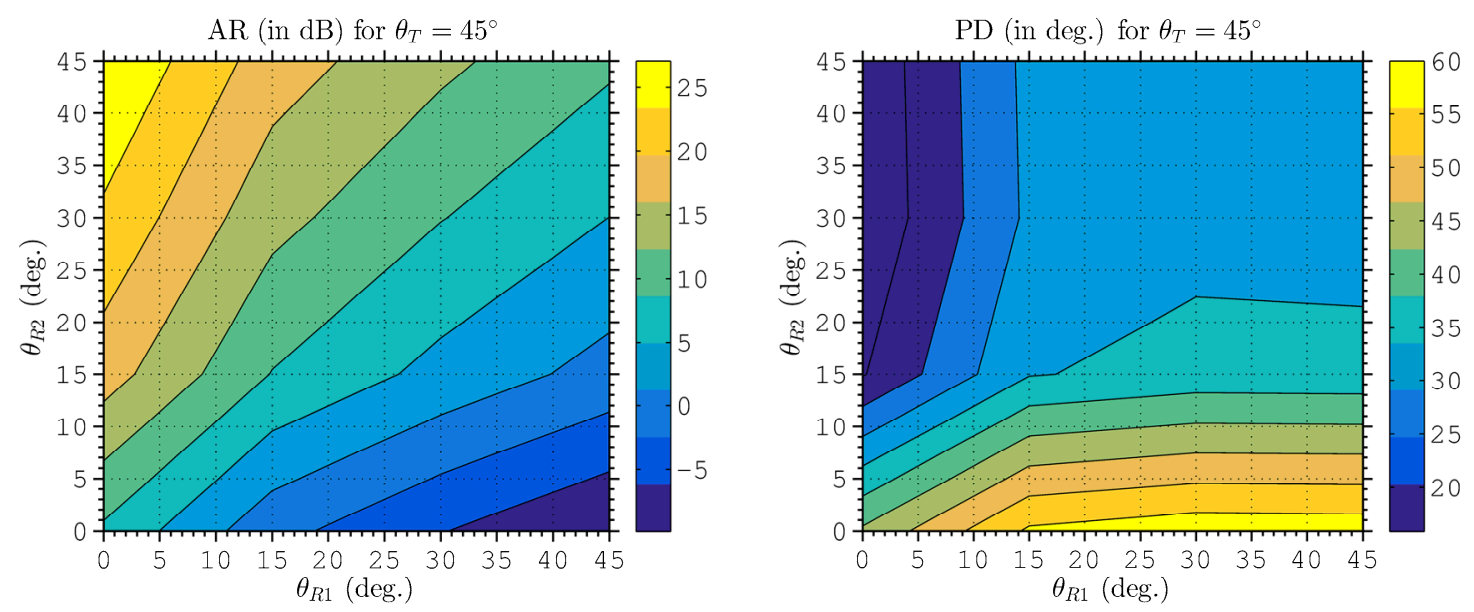

Fig. 15. Amplitude ratio and phase difference response for a transmitter TCA with $\theta_{T}=45^{\circ}$ in respect to the tilt angle of receivers. 


\section{CONCLUSION}

We have introduced a new pseudo-analytical formulation to model tilted-coil antennas tools in anisotropic formations. The combination of well-known closed-form solutions of Maxwell's equations in cylindrical coordinates with an efficient PML allows us to express the fields in radially-layered Earth formations as a sum of discrete modes. The TCA source is expanded in terms of modal fields, and the fields are found using axial mode-matching. We showed numerical results to validate the method and illustrate its ability to analyze directional well-logging tools. As a future work, this technique will be extended to analyze the fields in curved wells.

\section{ACKNOWLEDGMENT}

The first author acknowledges support from the Brazilian Agencies CNPq and CAPES through the doctoral fellowships 140236/2013-9, 141847/2016-6 and 000316/2015-06.

\section{REFERENCES}

[1] Y.-K. Hue and F. Teixeira, "FDTD simulation of MWD electromagnetic tools in large-contrast geophysical formations," IEEE Trans. Magn., vol. 40, pp. 1456-1459, Mar. 2004.

[2] Y.-K. Hue, "Analysis of electromagnetic well-logging tools," Ph.D. dissertation, The Ohio State University, Columbus, 2006.

[3] H. O. Lee and F. L. Teixeira, "Cylindrical FDTD analysis of LWD tools through anisotropic dipping-layered earth media," IEEE Trans. Geosci. Remote Sens., vol. 45, pp. 383-388, 2007.

[4] M. S. Novo, L. C. da Silva, and F. L. Teixeira, "Three-dimensional finite-volume analysis of directional resistivity logging sensors," IEEE Trans. Geosci. Remote Sens., vol. 48, no. 3, pp. 1151-1158, Mar. 2010.

[5] - "A comparative analysis of krylov solvers for three-dimensional simulations of borehole sensors," IEEE Geosci. Remote Sens. Lett.,vol. 8, no. 1, pp. 98-102, Jan. 2011.

[6] J. Lovell and W. Chew, "Response of a point source in a multicylindrically layered medium," IEEE Trans. Geosci. Remote Sens., vol. 25, pp. 850-858, Nov. 1987.

[7] T. Hagiwara et al., "Effects of mandrel, borehole, and invasion for tilt-coil antennas," in SPE 78th Ann. Tech. Conf. Exhibit., 5-8 Oct. 2003.

[8] Y.-K. Hue and F. L. Teixeira, "Analysis of tilted-coil eccentric borehole antennas in cylindrical multilayered formations for well-logging applications," IEEE Trans. Ant. Prop., vol. 54, pp. 1058-1064, 2006.

[9] G. S. Liu, F. L. Teixeira, and G. J. Zhang, "Analysis of directional logging tools in anisotropic and multieccentric cylindrically-layered earth formations," IEEE Trans. Antennas Propag., vol. 60, pp. 318-327, Jan. 2012.

[10] H. Wang, P. So, S. Yang, W. J. R. Hoefer, and H. Du, "Numerical modeling of multicomponent induction well-logging tools in the cylindrically stratified anisotropic media," IEEE Trans. Geosci. Remote Sens., vol. 46, no. 4, pp. 11341147, Apr. 2008.

[11] W. C. Chew et al., "Diffraction of axisymmetric waves in a borehole by bed boundary discontinuities," Geophys., vol. 49, pp. 1586-1595, 1984.

[12] Q.-H. Liu, "Electromagnetic field generated by an off-axis source in a cylindrically layered medium with an arbitrary number of horizontal discontinuities," Geophys., vol. 58, pp. 616-625, 1993.

[13] W. C. Chew, Waves and Fields in Inhomogeneous Media. New York, NY: IEEE Press, 1995.

[14] Y.-K. Hue and F. Teixeira, "Numerical mode-matching method for tilted-coil antennas in cylindrically layered anisotropic media with multiple horizontal beds," IEEE Trans. Geosci. Remote Sens., vol. 45, pp. 2451-2462, 2007.

[15] H. Moon, B. Donderici, F. L. Teixeira, "Stable evaluation of Green's functions in cylindrically stratified regions with uniaxial anisotropic layers," J. Comp. Phys., vol. 325, pp. 174-200, 2016.

[16] G. S. Rosa, J. R. Bergmann, and F. L. Teixeira, "A robust mode-matching algorithm for the analysis of triaxial welllogging tools in anisotropic geophysical formations," IEEE Trans. Geosci Remote Sens., submitted, 2016.

[17] L. M. Delves and J. N. Lyness, "A numerical method for locating the zeros of an analytic function," Mathematics of Computation, vol. 21, no. 100, pp. 543-560, Oct. 1967.

[18] J. W. Brown and R. V. Churchill, Complex Variables and Applications, 7th ed. New York, NY, USA: McGrawHill, 2004.

[19] G. S. Rosa and J. R. Bergmann, "Pseudo-analytical modeling for the electromagnetic propagation in stratified cylindrical structures," IEEE Antennas Wireless Propag. Lett., vol. 15, pp. 344-347, 2016.

[20] W. C. Chew and W. H. Weedon, “A 3D perfectly matched medium from modified Maxwell's equations with stretched coordinates," Microw. Opt. Tech. Lett., vol. 7, pp. 599-604, 1994.

[21] F. L. Teixeira and W. C. Chew, "Complex space approach to perfectly matched layers: a review and some new developments," Int. J. Num. Model., vol. 13, pp. 441-455, 2000.

[22] P. Bienstman et al., "Analysis of cylindrical waveguide discontinuities using vectorial eigenmodes and perfectly matched layers," IEEE Trans. Microw. Theory Techn., vol. 49, pp. 349-354, Feb. 2001. 
[23] P. Bienstman and R. Baets, "Advanced boundary conditions for eigenmode expansion models," Optical and Quantum Electronics, vol. 34, no. 5, pp. 523-540, May. 2002.

[24] R. F. Harrington, Time-harmonic electromagnetic fields. New York, NY, USA: McGraw-Hill, 1961.

[25] V. H. Rumsey, "Reaction concept in electromagnetic theory," Phys. Rev., vol. 94, pp. 1483-1491, Jun. 1954.

[26] K. Zaki, S.-W. Chen, and C. Chen, "Modeling discontinuities in dielectric-loaded waveguides," IEEE Trans. Microw. Theory Techn., vol. 36, no. 12, pp. 1804-1810, Dec. 1988.

[27] D. M. Pozar, Microwave Engineering, 3rd ed. Hoboken, NJ, USA: John Wiley \& Sons, Inc., 2005.

[28] M. Abramowitz and I. A. Stegun, Handbook of Mathematical Functions: With Formulas, Graphs, and Mathematical Tables. New York, NY, USA: Dover Publications, 1964. 